\title{
Polymorphisms in base excision repair genes and thyroid cancer risk
}

\author{
LUÍS S. SANTOS ${ }^{1,7^{*}}$, SANDRA C. BRANCO ${ }^{1 *}$, SUSANA N. SILVA ${ }^{1}$, \\ ANA PAULA AZEVEDO ${ }^{2,3}$, OCTÁVIA M. GIL ${ }^{1,4}$, ISABEL MANITA ${ }^{5}$, TERESA C. FERREIRA ${ }^{6}$, \\ EDWARD LIMBERT ${ }^{6}$, JOSÉ RUEFF ${ }^{1}$ and JORGE F. GASPAR ${ }^{1}$
}

\begin{abstract}
Departments of ${ }^{1}$ Genetics and ${ }^{2}$ Biochemistry, Faculty of Medical Sciences, Universidade Nova de Lisboa, Lisbon; ${ }^{3}$ Department of Clinical Pathology, Hospital São Francisco Xavier, Lisbon; ${ }^{4}$ Department of Radiological Protection and Nuclear Safety, Nuclear and Technological Institute, Sacavém; ${ }^{5}$ Unit of Endocrinology, Hospital Garcia de Orta, Almada; ${ }^{6}$ Department of Nuclear Medicine, Portuguese Oncology Institute of Lisbon, Lisbon; ${ }^{7}$ Health Sciences Institute, Universidade Católica Portuguesa, Viseu, Portugal
\end{abstract}

Received March 19, 2012; Accepted June 8, 2012

DOI: 10.3892/or.2012.1975

\begin{abstract}
Thyroid cancer (TC) is the most frequent endocrine malignancy, accounting however for only $1-2 \%$ of all human cancers, and the best-established risk factor for TC is radiation exposure, particularly during childhood. Since the BER pathway seems to play an important role in the repair of DNA damage induced by IR and other genotoxicants, we carried out a hospital-based case-control study in order to evaluate the potential modifying role of 6 BER polymorphisms on the individual susceptibility to non-familial TC in 109 TC patients receiving iodine-131, and 217 controls matched for age ( \pm 2 years), gender and ethnicity. Our results do not reveal a significant involvement of XRCC1 Arg194Trp and Arg399Gln, OGG1 Ser326Cys, APEX1 Asp148Glu, MUTYH Gln335His and PARP1 Val762Ala polymorphisms on the individual susceptibility towards TC, mostly in aggreement with the limited available evidence. By histological stratification analyis, we observed that the association between the presence of heterozygozity in the MUTYH Gln335His polymorphism and TC risk almost reached significance for the papillary subtype of TC. This was the first time that the putative association between this polymorphism and TC susceptibility was evaluated. However, since the sample size was modest, the possibility of a type I error should not be excluded and this result should, therefore, be interpreted with caution.
\end{abstract}

Correspondence to: Dr Jorge Francisco Gaspar, Department of Genetics, Faculty of Medical Sciences, Universidade Nova de Lisboa, Rua da Junqueira 100, 1349-008 Lisbon, Portugal

E-mail: jorge.gaspar@fcm.unl.pt

${ }^{*}$ Contributed equally

Key words: SNPs polymorphisms, DNA base excision repair, thyroid cancer, cancer susceptibility
More in depth studies involving larger populations should be pursued in order to further clarify the potential usefulness of the MUTYH Gln335His genotype as a predictive biomarker of susceptibility to TC and the role of the remaining BER polymorphisms on TC susceptibility.

\section{Introduction}

Thyroid cancer (TC) is the most frequent endocrine malignancy, accounting however for only $1-2 \%$ of all human cancers. In contrast with most malignancies, increasing incidence rates have been consistently reported worldwide over recent decades, although mortality due to TC does not seem to follow this trend (1). TC arises mostly from the epithelial elements of the gland and is usually classified according to histological and clinical criteria (1). Papillary and follicular thyroid carcinomas are the most common histological varieties, with papillary TC representing about $85 \%$ of all cases. TC is unusual during childhood but incidence increases with age. Also, women are afflicted three times as often as men, particularly during reproductive years (1). Overall prognosis is generally good, except for undifferentiated (anaplastic) carcinomas, which account for $<5 \%$ of TC cases (1).

TC etiology remains an obscure subject: inherited familial factors such as germline mutations seem to be responsible for only a minor percentage of TC cases $(2,3)$. Hormonal and dietary factors (e.g. iodine intake) have also been suggested to be implicated in the etiology of the disease but evidence is limited and inconsistent or, in the best of cases, limited to specific subtypes of TC $(1,2)$. Clearly, the best-established risk factor for $\mathrm{TC}$ is radiation exposure, particularly during childhood: risk of TC is increased after exposure to ionizing radiation (IR) doses as low as $0.1 \mathrm{~Gy}$ and is both dose- and agedependent, being maximal about 20-30 years after exposure $(1,2)$. This long latency period between radiation exposure and TC diagnosis is expected since IR induces mostly recessive mutations, therefore, requiring a second event for tumor initiation (4). Even so, radiation exposure is estimated to account for 
$<10 \%$ of all TC, suggesting that other risk factors could also have a relevant impact in the etiology of this malignancy.

Since TC risk and frequency is significantly higher in relatives (particularly first-degree) of TC patients compared to the general population $(2,3,5)$, it is possible that highprevalence, low-penetrance genetic variations could also constitute predisposing factors for TC, specially when considered together with exposure to environmental risk factors such as IR. Germ-line DNA polymorphisms in genes involved in regulation of apoptosis and control of the cell cycle (e.g. TP53), in kinase-dependent signaling pathways (e.g. RET), in endobiotic or xenobiotic metabolism (e.g. GST and CYP superfamilies), in hormonal and iodine metabolism (e.g. $T G$ ) and in DNA repair (among others) have been associated with interindividual differences in TC risk (reviewed in refs. 3, 5). Also, in the first genome-wide association study ever performed on TC, two polymorphisms located near the FOXE1 (TTF-2) and NKX2-1 (TTF-1) genes (which encode for thyroid-specific transcription factors) were identified as strong genetic risk markers of sporadic papillary TC in European populations (6).

IR is considered mutagenic since it can cause damage to DNA either directly, introducing single and double-strand breaks (DSB) to the DNA helix, or indirectly, promoting the radiolysis of water to yield radicalar DNA-reactive species that cause oxidative base damage (4). Such DNA lesions can activate specific checkpoints, triggering cell cycle arrest, in order to allow for DNA damage repair or, ultimately, inducing apoptotic cell death. Cell replication in the presence of genetic errors can cause irreversible mutations, leading to malignant transformation (7). In line with this, significant increase in DNA damage was found in patients with TC (8), suggesting that DNA repair mechanisms are important in correcting DNA damage and that defective DNA repair capacity might contribute to the risk of TC.

Base excision repair (BER) and DSB repair pathways are especially important in re-establishing DNA integrity after irradiation $(7,9)$. The latter can be accomplished through homologous recombination, in which the double helix of the homologous, undamaged partner DNA molecule is used as a model, or through non-homologous end-joining repair, which involves direct ligation of the two double-strand-break ends $(7,9)$. The BER pathway, in turn, is the main pathway responsible for recognizing, excising, and repairing small chemical alterations of a single base (e.g. oxidative DNA damage) caused by free radicals formed endogenously or after exposure to exogenous agents such as IR $(7,9,10)$. Briefly, BER is a multistep process involving several proteins, such as 8-oxoguanine DNA glycosylase (OGG1), APEX nuclease (multifunctional DNA repair enzyme) 1 (APEX1), X-ray repair complementing defective repair in Chinese hamster cells 1 (XRCC1) or poly (ADP-ribose) polymerase (PARP1), that act via a number of coordinated sequential 'cut-and-patch'-type reactions to detect the lesion, remove the single damaged base, and fill in the resulting single-stranded gap using the intact complementary strand as template $(7,9-11)$.

As such, DNA repair polymorphisms that modulate the DNA repair capacity may contribute to individual susceptibility to DNA damaging agents and, hence, modify cancer risk. In fact, polymorphic variants in virtually all DNA repair pathways have been associated with susceptibility for several types of cancer (7), including TC: regarding the latter, for example, polymorphisms in DNA repair genes such as ERCC2 (12) (NER pathway), Ku80 (13) (NHEJ pathway), XRCC3 (14), BRCA1 (15) and possibly RAD51 (14) (HR pathway) have been associated with TC risk (reviewed in refs. 3,5,9,16). Considering the BER pathway, in particular, several of the genetic variants identified in the XRCC1, OGG1, MUTYH, $A P E X 1$ and PARP1 genes have been reported to be associated with individual susceptibility to a variety of cancers, including breast, lung, colorectal and skin cancers $(7,10,11)$. Some of these polymorphisms also seem to affect IR sensitivity $(17,18)$. However, only a very limited number of studies have been published focusing on a possible role of BER pathway SNPs on TC susceptibility, mostly with negative or inconclusive results, except for XRCC1 polymorphisms (reviewed in refs. 3,9,16).

Since the BER pathway seems to play an important role in the repair of DNA damage induced by IR (the most well-known risk factor for TC) and other genotoxicants, we carried out an hospital based case-control study in a Caucasian Portuguese population in order to evaluate the potential modifying role of 6 BER polymorphisms on the individual susceptibility to non-familial TC.

\section{Materials and methods}

Study subjects. One hundred and nine TC patients receiving iodine-131 treatment at the Department of Nuclear Medicine of the Portuguese Oncology Institute of Lisbon were recruited. None of the patients enrolled in this study presented familiar history of TC. The TC diagnosis was confirmed histologically. For each case (except for one), two controls were recruited at São Francisco Xavier Hospital (Department of Laboratory Medicine), matched for age ( \pm 2 years), gender and ethnicity. Inclusion criteria for the control group included absence of cancer or thyroid disease and no familiar history of thyroid pathology. All study subjects were Caucasian, Portuguese, with Portuguese ascendants and no previous history of oncologic disease.

The anonymity of the patients and control population was guaranteed. Both patient and control participants groups were submitted to a questionnaire, performed by trained interviewers, in order to collect information on demographic characteristics, family history of cancer, lifestyle habits (smoking and alcohol drinking) and exposure to IR. In respect to smoking habits, former smokers were considered as nonsmokers if they gave up smoking either 2 years before TC diagnosis or 2 years before the inclusion date as control.

For all eligible participants, written informed consent was obtained prior to blood withdrawal. The response rate was $>95 \%$ for cases and controls. This study was approved by the ethics board of the involved institutions.

DNA extraction. Peripheral blood samples from all study participants were collected into $10-\mathrm{ml}$ heparinized tubes and kept thereafter at $-80^{\circ} \mathrm{C}$. Genomic DNA was extracted from $250 \mu \mathrm{l}$ of each blood sample using a commercially available kit (QIAamp ${ }^{\circledR}$ DNA mini kit; Qiagen), according to the manufacturer's instructions. All DNA samples were stored at $-20^{\circ} \mathrm{C}$ until further analysis. 
Table I. Selected SNP's and detailed information on the corresponding base and amino acid exchanges as well as minor allele frequency.

\begin{tabular}{|c|c|c|c|c|}
\hline Gene & Region & Codon & Base (amino acid) exchange & Minor allele frequency, $\operatorname{MAF}(\%)^{\mathrm{a}}$ \\
\hline \multirow[t]{2}{*}{$X R C C 1$} & Exon 6 & 194 & $\mathrm{C} \rightarrow \mathrm{T}(\mathrm{Arg} \rightarrow \mathrm{Trp})$ & 13.1 \\
\hline & Exon 10 & 399 & $\mathrm{G} \rightarrow \mathrm{A}(\mathrm{Arg} \rightarrow \mathrm{Gln})$ & 26.6 \\
\hline$O G G 1$ & Exon 6 & 326 & $\mathrm{C} \rightarrow \mathrm{G}(\mathrm{Ser} \rightarrow \mathrm{Cys})$ & 29.9 \\
\hline$A P E X 1$ & Exon 5 & 148 & $\mathrm{~T} \rightarrow \mathrm{G}(\mathrm{Asp} \rightarrow \mathrm{Glu})$ & 44.0 \\
\hline MUTYH & Exon 12 & 335 & $\mathrm{G} \rightarrow \mathrm{C}(\mathrm{Gln} \rightarrow \mathrm{His})$ & 31.9 \\
\hline PARPl & Exon 17 & 762 & $\mathrm{~T} \rightarrow \mathrm{C}(\mathrm{Val} \rightarrow \mathrm{Ala})$ & 24.4 \\
\hline
\end{tabular}

aAccording to http://www.ncbi.nlm.nih.gov/projects/SNP/.

Single nucleotide polymorphisms (SNP's) selection. Publicly available on-line databases such as NCBI (http://www.ncbi. nlm.nih.gov/snp/), GeneCards (http://www.genecards.org) and SNP500Cancer (http://variantgps.nci.nih.gov/cgfseq/pages/ snp500.do) were used to search for SNPs reported to date on genes coding for DNA repair proteins of the BER pathway. Eligible SNPs had to be located in a coding region, give rise to an amino acid change (non-synonymous) and exhibit a minor allele frequency $>0.1$ in Caucasian populations According to these criteria, 6 common nsSNPs were selected for genotyping: Arg194Trp [reference SNP no. (rs) 1799782] and Arg399Gln (rs25487) for XRCC1; Ser326Cys (rs1052133) for $O G G 1$; Asp148Glu (rs1130409) for APEX1; Gln335His (rs3219489) for MUTYH and Val762Ala (rs1136410) for PARPI (Table I).

Genotyping. PCR-RFLP-XRCCl Arg194Trp, XRCC1 Arg399Gln and $O G G 1$ Ser326Cys polymorphisms were genotyped by polymerase chain reaction (PCR) followed by restriction fragment length polymorphism (RFLP). Primer design and PCR conditions were optimized in order to achieve the best possible results: OligoAnalyzer 3.1 (http://eu.idtdna. com/analyzer/Applications/OligoAnalyzer/) was used to determine melting temperatures, GC contents, hairpins, and dimmer formation. Basic local alignment search tool (BLAST) resource (http://blast.ncbi.nlm.nih.gov/Blast.cgi) was used to confirm low similarity with other human sequences. All PCR reactions were performed in a $50 \mu \mathrm{l}$ final volume, comprising $3 \mu \mathrm{l}$ genomic DNA, 1X ImmoBuffer, $2.5 \mathrm{mM} \mathrm{MgCl}_{2}$, $0.8 \mathrm{mM}$ of each dNTP (Bioline), $0.6 \mu \mathrm{M}$ of each primer (Stabvida), $0.909 \mu 1 \mathrm{DMSO}$ and $0.75 \mathrm{U}$ of Immolase (Bioline). Amplification was accomplished in a GeneAmp® PCR system 2700 thermal cycler (Applied Biosystems): the initial enzyme activation step $\left(95^{\circ} \mathrm{C}, 7 \mathrm{~min}\right)$ was ensued by a variable number of amplification cycles consisting of denaturation $\left(94^{\circ} \mathrm{C}\right.$, $30 \mathrm{sec}$ ), annealing (variable temperature, $30 \mathrm{sec}$ ) and extension $\left(72^{\circ} \mathrm{C}, 30 \mathrm{sec}\right)$; amplification was concluded with a final extension step at $72^{\circ} \mathrm{C}$ for $10 \mathrm{~min}$. Primer sequences, annealing temperatures, number of amplification cycles (PCR conditions) and PCR product size (bp) specific for both XRCC1 polymorphisms have been described elsewhere (19). Specific primer sequences (forward: 5'-ggt ggc cct aaa gga ctc tcc-3'; reverse: $5^{\prime}$-cca tcc tta gcg ctg tct ccc-3') and PCR conditions (35 PCR cycles, with an annealing temperature of $64^{\circ} \mathrm{C}$ ) were used for the determination of $O G G 1$ Ser326Cys genotype, yelding a 456 bp PCR product.

After amplification, $10 \mu \mathrm{l}$ of each PCR product were incubated with 5 units of an appropriate restriction enzyme and $1.25 \mu \mathrm{l}$ of the $10 \mathrm{X}$ buffer recommended by the enzyme manufacturer, to a final volume of $12.5 \mu \mathrm{l}$. The choice of the restriction enzyme and reaction conditions were optimized as follows: to genotype the XRCC1 Arg194Trp polymorphism, the previously amplified fragment was digested with $M s p I$ (Fermentas) for $2 \mathrm{~h}$ at $37^{\circ} \mathrm{C}$. The exact same conditions were applied to the restriction analysis of the XRCC1 Arg399GIn polymorphism while for the $O G G 1$ Ser326Cys polymorphism the digestion was performed overnight at $37^{\circ} \mathrm{C}$ with the SatI restriction enzyme (Fermentas).

After restriction enzyme inactivation $\left(20 \mathrm{~min}\right.$ at $\left.65^{\circ} \mathrm{C}\right)$, the resulting digested fragments were electrophoresed in an ethidium bromide-stained $4 \%$ agarose gel and visualized under ultraviolet light. HyperLadder V (Bioline) was used as molecular marker. The expected digestion pattern for each genotype of the XRCC1 Arg194Trp and XRCC1 Arg399Gln polymorphisms has been previously described (19). For the OGG1 Ser326Cys polymorphism, fragment length after SatI digestion was $456 \mathrm{bp}$ for the $\mathrm{C} / \mathrm{C}$ genotype, 456, 258, $198 \mathrm{bp}$ for the $\mathrm{C} / \mathrm{G}$ genotype and 258, 198 bp for the $\mathrm{G} / \mathrm{G}$ genotype.

All the genotype determinations were carried out twice in independent experiments and inconclusive samples were re-analysed.

Real-time PCR-APEX1 Asp148Glu, MUTYH Gln335His and $P A R P 1$ Val762Ala polymorphisms were genotyped by real-time PCR, using the TaqMan fluorogenic 5' nuclease assay (Applied Biosystems). In order to assure uniformity in genomic DNA content $\left(2.5 \mathrm{ng} . \mu \mathrm{l}^{-1}\right)$ in all samples analyzed through this method, DNA was pre-quantified by fluorimetry with the Quant-iT ${ }^{\mathrm{TM}}$ PicoGreen ${ }^{\circledR}$ dsDNA Assay kit (Invitrogen) and a Zenyth 3100 plate reader (Anthos Labtech Instruments), according to the manufacturer's instructions.

Amplification was carried out in a 7300 Real-Time PCR System thermal cycler (Applied Biosystems), with 96-well microplates containing $10 \mathrm{ng}$ of genomic DNA, 1X SNP genotyping assay mix (APEX1 assay ID: C__8921503_10; MUTYH assay ID: $\mathrm{C}_{-} 27504565 \_10 ; P A R P 1$ assay ID: C__1515368_1_) and 1X TaqMan Universal PCR Master mix per well (final volume, $10 \mu \mathrm{l} /$ well). Initial enzyme activation $\left(10 \mathrm{~min}\right.$, at $\left.95^{\circ} \mathrm{C}\right)$ was ensued by 40 cycles of denaturation 
Table II. General characteristics for the TC cases $(n=109)$ and control population $(n=217)$.

\begin{tabular}{lccc}
\hline Characteristics & Cases, n (\%) & Controls, n (\%) & P-value $^{\mathrm{c}, \mathrm{d}}$ \\
\hline Gender & & & \\
Female & $92(84.4)$ & $185(85.3)$ & 0.839 \\
Male & $17(15.6)$ & $32(14.7)$ & \\
Age & & & \\
$\leq 30$ & & $9(4.1)$ & 0.997 \\
$31-49$ & $4(3.7)$ & $77(35.5)$ & \\
$50-69$ & $39(35.8)$ & $104(47.9)$ & \\
$\geq 70$ & $52(47.7)$ & $27(12.4)$ & \\
Smoking habits & $14(12.8)$ & & \\
Non-smoker & $97(89)$ & $177(82.3)$ & 0.117 \\
Smoker & $12(11)$ & $38(17.7)$ & \\
Missing & 0 & $2(0.6)$ & \\
\end{tabular}

${ }^{\mathrm{a}} \mathrm{Age}$ at diagnosis for cases. ${ }^{\mathrm{b}} \mathrm{Age}$ at enrollment for each of the casematched controls. ${ }^{\mathrm{c} S e e}$ Materials and methods; ${ }^{\mathrm{d}}$ cases vs. control group.

$\left(15 \mathrm{sec}\right.$ at $\left.92^{\circ} \mathrm{C}\right)$ and probe annealing/extension $\left(1 \mathrm{~min}\right.$ at $\left.60^{\circ} \mathrm{C}\right)$. Allelic discrimination was then performed by measuring fluorescence emitted by both VIC and FAM dyes in each well $(60 \mathrm{sec})$ and computing the results into the System SDS software version 1.3.1. All reactions were performed twice in independent experiments and repeated for all inconclusive samples.

Statistical analysis. Exact probability tests available in Mendel software (V5.7.2) were used to analyze the Hardy-Weinberg frequencies for XRCC1, OGG1, APEX1, MUTYH and PARP1 alleles in patient and control populations, considered separately (20).

The differences in genotype frequency and smoking status between patient and control populations were evaluated by the Chi-square test. The normality of continuous variables, such as age, and the homogeneity of variances were analyzed with Kolmogorov-Smirnov and Levene tests, respectively. The Student's t-test was used in the statistical analysis of the homogeneity of age distribution between patient and control populations, while the Chi-square test was applied to the analysis of the homogeneity of gender distribution.

When assessing the genetic effects, logistic regression analysis was performed to determine the crude and adjusted odds ratio (OR) and the corresponding 95\% confidence intervals (CI). Adjusted OR calculations took into account gender, age at diagnosis ( $\leq 30,31-49,50-69$ and $\geq 70$ years) and smoking habits (smokers/non-smokers), with female gender, lower age group and non-smokers being considered as the

Table III. Genotype frequencies for each of the selected BER polymorphisms in TC case and control populations.

\begin{tabular}{|c|c|c|c|c|c|c|}
\hline \multirow[b]{2}{*}{ Polymorphism } & \multirow[b]{2}{*}{$\begin{array}{l}\text { Reference } \\
\text { form }^{\mathrm{a}}\end{array}$} & \multirow[b]{2}{*}{$\mathrm{n}$} & \multicolumn{3}{|c|}{ Genotype frequency } & \multirow[b]{2}{*}{ P-value } \\
\hline & & & $\begin{array}{c}\text { Reference } \\
\text { homozygote, } \mathrm{n}(\%)\end{array}$ & Heterozygote, n (\%) & $\begin{array}{c}\text { Variant } \\
\text { homozygote, n }(\%)\end{array}$ & \\
\hline \multicolumn{7}{|c|}{ XRCC1 (Arg194Trp) } \\
\hline Control group & Arg & 217 & $196(90.3)$ & $21(9.7)$ & $0(0.0)$ & 0.109 \\
\hline Case group & & 108 & $98(90.7)$ & $87.4)$ & $2(1.9)$ & \\
\hline \multicolumn{7}{|c|}{ XRCC1 (Arg399Gln) } \\
\hline Control group & Arg & 217 & $87(40.1)$ & $105(48.4)$ & $25(11.5)$ & 0.911 \\
\hline Case group & & 109 & $46(42.2)$ & $50(45.9)$ & $13(11.9)$ & \\
\hline \multicolumn{7}{|c|}{ OGG1 (Ser326Cys) } \\
\hline Control group & Ser & 217 & $139(64.1)$ & $78(35.9)$ & $0(0.0)$ & 0.335 \\
\hline Case group & & 108 & $75(69.4)$ & $33(30.6)$ & $0(0.0)$ & \\
\hline \multicolumn{7}{|c|}{ APEX1 (Asp148Glu) } \\
\hline Control group & Asp & 217 & $56(25.8)$ & $103(47.5)$ & $58(26.7)$ & 0.810 \\
\hline Case group & & 109 & $31(28.4)$ & $52(47.7)$ & $26(23.9)$ & \\
\hline \multicolumn{7}{|c|}{ MUTYH (Gln335His) } \\
\hline Control group & His & 216 & $106(49.1)$ & $102(47.2)$ & $8(3.7)$ & 0.195 \\
\hline Case group & & 109 & $64(58.7)$ & $40(36.7)$ & $5(4.6)$ & \\
\hline \multicolumn{7}{|c|}{ PARP1 (Val762Ala) } \\
\hline Control group & Val & 216 & $168(77.8)$ & $48(22.2)$ & $0(0.0)$ & 0.270 \\
\hline Case group & & 108 & $78(72.2)$ & $30(27.8)$ & $0(0.0)$ & \\
\hline
\end{tabular}

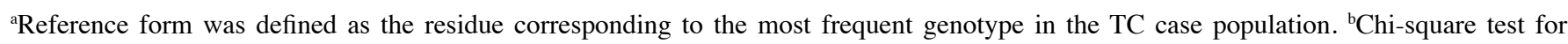
distribution of genotypic frequencies. 
Table IV. Comparison of genotypic frequencies observed in the control group of this study with those previously published in the literature for Caucasian populations.

\begin{tabular}{|c|c|c|c|c|}
\hline \multirow[t]{2}{*}{ Polymorphism } & \multicolumn{3}{|c|}{ Genotype frequency } & \multirow[t]{2}{*}{ Population } \\
\hline & Arg/Arg, n (\%) & $\operatorname{Trp} / \operatorname{Arg}, \mathrm{n}(\%)$ & $\operatorname{Trp} / \operatorname{Trp}, \mathrm{n}(\%)$ & \\
\hline \multirow[t]{3}{*}{ XRCC1 (Arg194Trp) } & $196(90.3)$ & $21(9.7)$ & $0(0.0)$ & Caucasian Portuguese (study data) \\
\hline & $1377(88.5)$ & $177(11.4)$ & $2(0.1)$ & Caucasian Northern European $(n=1556)(21)$ \\
\hline & Arg/Arg, n (\%) & Arg/Gln, n (\%) & $\mathrm{Gln} / \mathrm{G} \ln , \mathrm{n}(\%)$ & \\
\hline \multirow[t]{3}{*}{ XRCC1 (Arg399Gln) } & $87(40.1)$ & $105(48.4)$ & $25(11.5)$ & Caucasian Portuguese (study data) \\
\hline & $645(41.6)$ & $728(47.0)$ & $176(11.4)$ & Caucasian Northern European $(n=1549)(21)$ \\
\hline & Ser/Ser, n $(\%)$ & Ser/Cys, n (\%) & Cys/Cys, n (\%) & \\
\hline \multirow[t]{3}{*}{ OGG1 (Ser326Cys) } & $139(64.1)$ & $78(35.9)$ & $0(0.0)$ & Caucasian Portuguese (study data) \\
\hline & $760(61.1)$ & $424(34.1)$ & $60(4.8)$ & Caucasian American ( $\mathrm{n}=1244)(22)$ \\
\hline & Asp/Asp, n (\%) & Asp/ Glu, n (\%) & Glu/Glu, n (\%) & \\
\hline \multirow[t]{3}{*}{ APEX1 (Asp148Glu) } & $56(25.8)$ & $103(47.5)$ & $58(26.7)$ & Caucasian Portuguese (study data) \\
\hline & $327(27.09)$ & $590(48.88)$ & $290(24.03)$ & Caucasian American ( $\mathrm{n}=1207)(22)$ \\
\hline & Gln/Gln, n (\%) & Gln/His, n (\%) & His/His, n (\%) & \\
\hline \multirow[t]{3}{*}{ MUTYH (Gln335His) } & $8(3.7)$ & $102(47.2)$ & $106(49.1)$ & Caucasian Portuguese (study data) \\
\hline & $29(5.3)$ & $235(43.0)$ & $283(51.7)$ & Caucasian Portuguese ( $\mathrm{n}=547$, only women) (23) \\
\hline & $\mathrm{Val} / \mathrm{Val}, \mathrm{n}(\%)$ & Val/Ala, n (\%) & Ala/Ala, n (\%) & \\
\hline \multirow[t]{2}{*}{ PARPl (Val762Ala) } & $168(77.8)$ & $48(22.2)$ & $0(0.0)$ & Caucasian Portuguese (study data) \\
\hline & $963(70.2)$ & $361(26.3)$ & $47(3.4)$ & Caucasian American $(n=1371)(22)$ \\
\hline
\end{tabular}

reference groups for each of these variables. For the purpose of these calculations, age at diagnosis for controls was the age at enrollment.

All analyses were performed using the Statistical Package for the Social Sciences for Windows 15.0 version (SPSS, Inc., Chicago IL, USA).

\section{Results}

This study comprised 109 TC patients and 217 age- and gendermatched controls. According to histological criteria, 71\% TC cases were classified as papillary TC (78 patients), $26 \%$ as follicular TC (28 patients) and 3\% as poorly differentiated TC (3 patients). Previous exposure to IR sources, with the exception for diagnostic medical X-rays, was denied by all cases.

The baseline characteristics (gender, age and smoking habits) of both case and control populations are listed in Table II. The case group included 17 male and 92 female patients, with an overall mean age of 53 years. In agreement with the gender distribution usually observed in this type of cancer, the frequency of females in the case group was significantly higher than the frequency of males. No significant difference was found between the case and control groups concerning age distribution $(\mathrm{P}=0.997)$, gender $(\mathrm{P}=0.839)$ or smoking habits $(\mathrm{P}=0.117)$.
The genotype frequencies of the 6 polymorphisms, in both TC cases and controls, are shown in Table III. The frequency of minor allele homozygotes for the OGG1 Ser326Cys and PARP1 Val762Ala polymorphisms was null both in case and control study groups. The same was observed for the XRCCI Arg194Trp polymorphism in the control group. The genotypic frequencies of the XRCC1 Arg399Gln, APEX1 Asp148Glu and $P A R P I$ Val762Ala polymorphisms in control and cancer groups were in agreement with the expectations of the HardyWeinberg law ( $\mathrm{P}>0.1$, exact probability test). The same was not observed, however, for the OGG1 Ser326Cys and MUTYH Gln335His polymorphisms in the control group nor for the XRCC1 Arg194Trp polymorphism in the cancer group.

The genotypic frequencies that were observed in the control group for the XRCC1 Arg194Trp and XRCC1 Arg399Gln polymorphisms are similar to those previously reported by Kiuru et al (21) for a Causasian Northern European population. The genotypic frequencies of the OGG1 Ser326Cys, APEX1 Asp148Glu and PARP1 Val762Ala polymorphisms are also in agreement with those reported in a larger study $(\mathrm{n}>1000)$ carried out in a Caucasian American population (22). As for the MUTYH Gln335His polymorphism, the genotypic frequencies reported are in agreement with those reported by Conde et al (23), for a similar Caucasian Portuguese population (Table IV). 


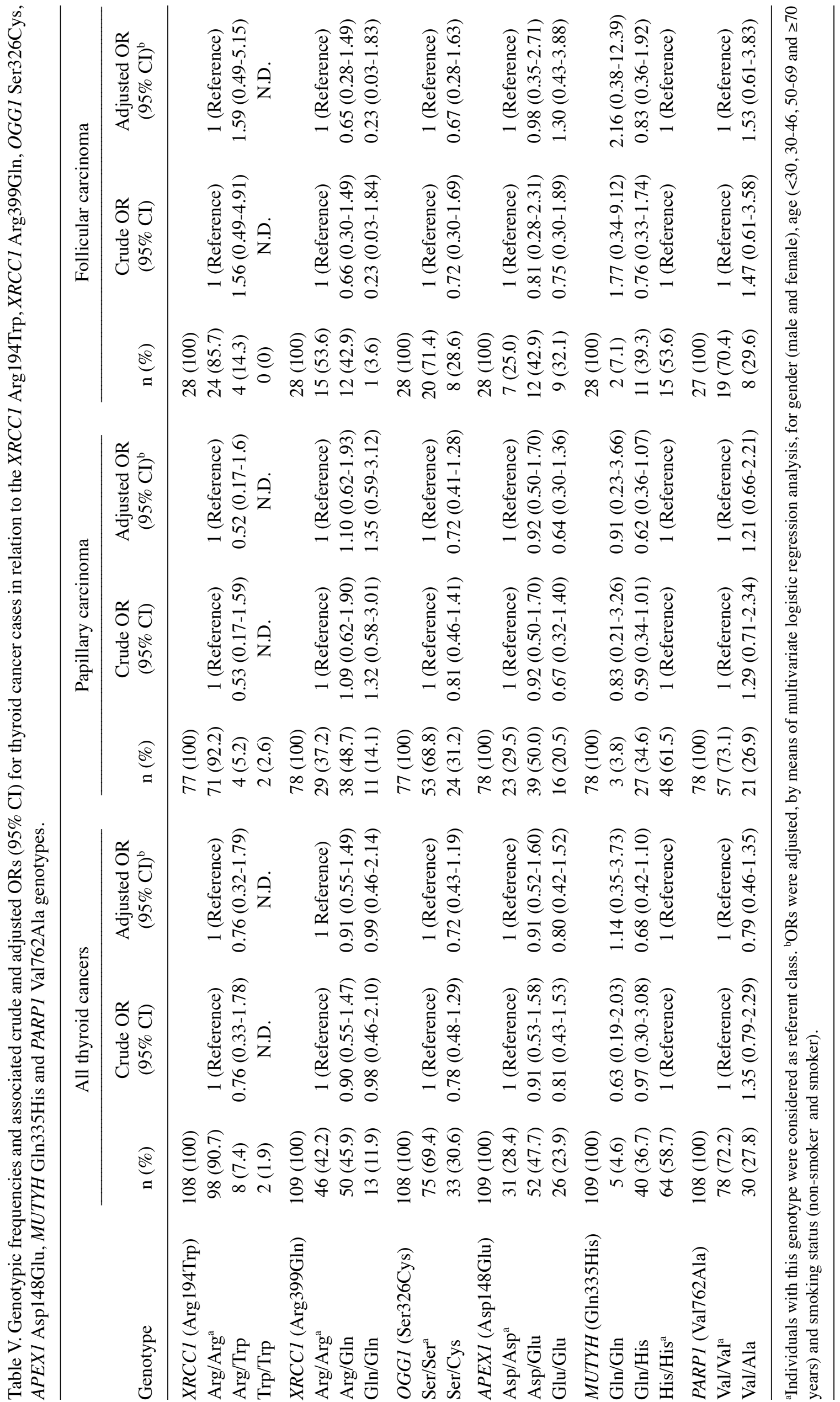


Since TC incidence is recurrently reported to be higher in women (which was also the predominant gender in our case group), we also compared genotypic frequencies according to gender among TC patients (in order to examine for any gender-specific genetic effect). The frequency of the different genotypes considered did not differ significantly with gender in TC patients (Chi-square test with Yates correction), even after TC stratification according to histological criteria (data not shown). For this reason, no further gender stratification of the population was considered in the statistical analysis.

As shown in Table V, no significant differences in genotypic frequencies were observed for any of the 6 selected polymorphisms, between cases and controls $(\mathrm{P}>0.05$, Chi-square test with Yates correction). Stratifying the data according to the histological classification of the tumors (papillary and follicular tumors) did not yield any significant results, irrespective of the polymorphism considered. Therefore, the results obtained concerning the genotype distributions for any of the selected polymorphisms in TC patients, when compared to controls, do not support an association between the presence of one genotype of any of these polymorphisms and individual susceptibility towards TC.

In addition, logistic regression analysis concerning the effect of the different polymorphisms on TC risk (adjusted for age at diagnosis, smoking habits and gender) was performed. No significant change in crude or adjusted OR was observed for any of the genotypes considered (Table V), further suggesting that none of the polymorphisms, individually considered, is associated with TC risk. Again, after stratifying data according to histological subtype, no significant change in crude or adjusted OR was revealed in either papillary or follicular TC subgroups. However, when considering only the papillary subtype of TC, OR values for the MUTYH Gln335His polymorphism almost reached significance: a borderline decrease in the risk of papillary TC was apparent for heterozygous $(\mathrm{Gln} / \mathrm{His}$ ) individuals (adjusted $\mathrm{OR}=0.6$; 95\% CI, 0.4-1.1; $\mathrm{P}=0.080$; Table V).

Finally, we evaluated whether the presence of variant forms in any of the selected polymorphisms was associated with early-age at diagnosis (i.e., predisposal to early development of the disease) or not. The observed results (data not shown) do not support this hypothesis since the age at TC diagnosis did not differ significantly with genotype, for any of the polymorphisms.

\section{Discussion}

In this study, we report the role of genetic polymorphisms XRCC1 Arg194Trp $(\mathrm{C} \rightarrow \mathrm{T})$ and Arg399Gln $(\mathrm{G} \rightarrow \mathrm{A}), O G G 1$ Ser326Cys $(\mathrm{C} \rightarrow \mathrm{G})$, APEX1 Asp148Glu $(\mathrm{T} \rightarrow \mathrm{G})$, MUTYH Gln335His $(\mathrm{G} \rightarrow \mathrm{C})$ and PARPl Val762Ala $(\mathrm{T} \rightarrow \mathrm{C})$ on the individual susceptibility for TC. The frequencies of the different genotypes observed in the control population are similar to those reported in other Caucasian populations.

$\mathrm{XRCC1}$ is a nuclear protein that, despite lacking any enzymatic activity, plays an important role in the efficient repair of SSBs and in the BER pathway: it acts as a scaffold protein that facilitates the recruitment of multiple DNA repair enzymes (such as Pol $\beta$, hOGG1, APEX1, PARP1 and LIG3) to lesion sites and coordinates the DNA damage repair response.
Arg194Trp and Arg399GIn are among the most extensively studied XRCC1 coding region SNPs. Both polymorphisms have been shown to alter the functional activity of the resulting protein in vitro and to interfere with cancer susceptibility: the 194Trp allele has been associated with increased protein function (hence, enhanced DNA repair capacity and lower sensitivity to genotoxicants) and decreased risk of certain cancers, particularly among smokers; on the contrary, the 399Gln allele is suggested to be associated with decreased DNA repair capacity and higher sensitivity to genotoxicants. Epidemiological evidence for an association between the Arg399Gln polymorphism and cancer susceptibility is weaker (often negative, inconsistent or conflicting) and associations in opposite directions depending on cancer type and smoking status have been suggested. Other gene-environment (e.g. drinking status or menopausal age) and gene-gene interactions (e.g. other DNA repair or chemical metabolizing enzymes) have also been reported for both SNPs. Numerous other well-powered studies and meta-analyses have not, however, confirmed these putative effects (reviewed in refs. 10,24). Overall, in face of the disparity in the results reported thus far, these amino acid substitutions appear to have only a modest (if any) impact on XRCC1 activity and cancer susceptibility (unless when considered together with other relevant environmental or genetic factors). The epidemiological studies performed thus far in respect to the contribution of these SNPs to TC susceptibility have yielded conflicting results. Our results do not support an association between TC risk and the XRCCI Arg399Gln polymorphism, in agreement with the majority of the studies (25-28). This hypothesis should not be ruled out, however, since two other groups have recently reported a protective effect for the $399 \mathrm{Gln}$ allele in well-powered cohorts: in both studies, the 399Gln allele was associated with decreased risk of differentiated TC $[\mathrm{OR}=0.70 ; 95 \% \mathrm{CI}$, $0.50-1.00 ; \mathrm{P}=0.049 ;(29)]$ or papillary TC $[\mathrm{OR}=0.70 ; 95 \% \mathrm{CI}$, 0.59-0.93; $\mathrm{P}=0.03$; (30)]. On the contrary, the 194Trp allele has been shown to increase the risk of developing differentiated TC [OR=1.4; 95\% CI, 0.9-2.1, for the heterozygous genotype (29); OR=1.85; 95\% CI, 1.11-3.07 (25)]. Furthermore, Chiang et al (25) observed that this genetic effect came primarily from the subjects with $\mathrm{LN}$ metastasis $(\mathrm{OR}=4.54$; 95\% CI, 2.11-9.79; $\mathrm{P}=0.0001$ ), and was also higher when the XRCCl and PARPI genotypes were considered together. The results in our study do not reveal a significant association between the XRCCl Arg194Trp variant genotype and TC risk in a Caucasian Portuguese population. It is possible that the differences in 194Trp allelic frequency between Caucasian and Asian populations obscured a possible association in our study. However, one must also recall that the 194Trp allele is often associated with enhanced DNA repair capacity, lower sensitivity to genotoxicants and decreased risk of other cancers (see above). In line with this, Sigurdson et al (28) observed, in a large cohort, that the minor allele of the XRCC1 Arg194Trp polymorphism was associated with decreased thyroid nodule risk $(\mathrm{OR}=0.5 ; 95 \%$ CI, 0.3-0.9; $\mathrm{P}=0.03)$, describing a similar pattern of association for a small number of papillary TC $(\mathrm{n}=25)(28)$. Also, in a small case-control study in a Korean population (27), the 194Trp heterozygous genotype was significantly associated with a decreased risk of papillary TC (OR=0.55; 95\% CI, 0.31-0.98). The results presented by Ho et al (29) and Chiang et al (25) 
should therefore be interpreted with caution. It is possible that the XRCC1 Arg194Trp polymorphism may have opposing effects depending on the disease development stage, on the presence of different environmental risk factors or on different genetic backgrounds among populations. Larger studies and/ or a meta-analysis are needed in order to further clarify the role of the XRCCl polymorphisms in TC susceptibility.

Concerning our data, the results do not reveal a significant involvement of $O G G 1$ Ser326Cys polymorphism on the individual susceptibility towards TC, since the genotype frequencies are similar in control and cancer patient populations (Table III), and no increase in TC risk was associated with this polymorphism (Table V). These results are in agreement with those recently reported by Garcia-Quispes et al (26) in the only other case-control study where the influence of the OGG1 Ser326Cys genotype on TC risk was also evaluated. The $O G G 1$ Ser326Cys polymorphism has been demonstrated to impair protein function (10) and, as such, has been widely evaluated in different case-control studies: the available evidence supporting an association with cancer susceptibility is scarce, however, with significant results being limited, mainly, to lung cancer $(11,31)$. Of notice, three recently performed meta-analysis (31-33) did not reveal any significant association between the $O G G 1$ Ser326Cys polymorphism and breast cancer risk: these studies, taken together, suggest a minor role for the OGG1 Ser326Cys polymorphism in endocrine cancer susceptibility (as is the case of TC).

The MUTYH protein is a BER glycosylase that is involved in the repair of oxidative DNA lesions such as 8-oxo-7,8-dihydro-2'-deoxyguanosine (8-oxo-dG), a stable oxidation product of guanine: if left unrepaired, 8-oxo-dG can mispair with adenine during DNA replication, leading to a $\mathrm{G}: \mathrm{C} \rightarrow \mathrm{T}: \mathrm{A}$ transversion. The MUTYH protein prevents these transversions by excising any adenine residue misincorporated in the newly synthesized DNA strand opposite to 8-oxo-dG, thus allowing a second opportunity for OGG1 to repair the lesion (34). MUTYH dysfunction may, therefore, be especially problematic for tumorigenesis in humans since there are no other mechanisms for repairing 8-oxo-dG/adenine mismatches. Accordingly, as excellently reviewed elsewhere (35), two germline missense mutations in the MUTYH gene that result in a catalytically compromised protein, Tyr165Cys and Gly382Asp, have been unequivocally demonstrated to be associated with a colorectal adenoma and carcinoma predisposition syndrome that is now referred to as MUTYHassociated polyposis (MAP): MAP phenotypically resembles the classic or the attenuated forms of familial adenomatous polyposis (FAP) but is transmitted as an autosomal recessive trait associated with inherited biallelic defects in the $M U T Y H$ gene. MAP tumours display an unusually high proportion of somatic $\mathrm{G}: \mathrm{C} \rightarrow \mathrm{T}: \mathrm{A}$ transversion mutations in the $A P C$ and $K$-ras genes, probably reflecting defective MUTYH protein activity and consequent failure to correct A:8-oxo-dG mispairs. It is worth mentioning that, as it has been firmly established for FAP patients, MAP patients may also be at increased risk of developing extra-colonic tumours such as TC: TC has sporadically been observed in MAP patients $(36,37)$ but, for the time being, evidence is insufficient to establish an association between MAP and TC. Besides the Tyr165Cys and Gly382Asp variants, many other MUTYH germline mutations and SNPs have been described to date, most of them rare and/or of 'uncertain pathogenicity' (35). MUTYH Gln335His is a common polymorphism located on exon 12 (35), more frequently detected in Japanese and Chinese than in European populations. The enzyme encoded by this variant has been demonstrated to have partially impaired glycosylase activity in vitro (compared to the wild-type, the variant enzyme was $34 \%$ less active in removing adenine from substrates containing an A:GO mismatch) (38) and could therefore contribute to cancer susceptibility. The possible association between the MUTYH Gln335His polymorphism and cancer risk has been extensively studied in Asian (mainly Japanese) and other populations: the $335 \mathrm{His}$ variant allele has been suggested to be associated with increased risk of colorectal cancer (mainly when limited to specific anatomical locations) $(39,40)$ and, less consistently, lung cancer (41). For the latter, however, existing evidence is conflicting or significant only when taking gene-gene interactions into account (42). To our knowledge, no significant correlation between the MUTYH Gln335His genotype and cancer risk has been described for any other type of cancer. Interestingly, an almost significant decrease in breast cancer risk (OR=0.80, 95\% CI, 0.59-1.07) in MUTYH Gln335His heterozygotes (Gln/His) was reported in a Portuguese Caucasian population (23). Also, in a bladder cancer susceptibility study, gene-gene interactions among BER polymorphisms (including MUTYH Gln335His) were observed in ever smokers (43), suggesting that BER genetic variation might contribute to cancer risk through gene-gene and geneenvironmental interactions. To the best of our knowledge, no clinical association studies have been performed thus far to evaluate the role of the MUTYH Gln335His polymorphism on TC susceptibility. The results reported here for the MUTYH Gln335His polymorphism suggest that it is not associated with TC risk (Table V). However, when considering only the papillary subtype of TC, OR values for the MUTYH Gln335His polymorphism almost reached significance: a borderline effect associated to a decreased risk of papillary TC was apparent for heterozygous (Gln/His) individuals (Table V). However, it should not be excluded that this effect could be related to sample size. This is the first time that the putative association between the MUTYH Gln335His polymorphism and TC susceptibility has been evaluated. The results reported here strongly suggest that its role in papillary TC susceptibility be further evaluated in a larger study population, in order to verify its potential usefulness as a predictive biomarker of genetic susceptibility to TC.

APEX1, the major human AP endonuclease, plays a central role in the BER pathway due to its ability to process abasic sites and other 3' DNA termini that may result, for example, from exposure to IR or direct attack by free radicals (11). The APEX1 Asp148Glu polymorphism is the most common and most studied non-synonymous APEXI coding region variant $(10,11)$. Despite the enzyme encoded by the variant form of this polymorphism has been demonstrated in vitro to possess no significant impact on endonuclease and DNA binding activities, its potential role on cancer susceptibility has been frequently studied: as expected, the largest and strongest body of evidence (including four recent GWAS on breast cancer susceptibility and a meta-analysis on both lung and aerodigestive tract cancers) suggests no association between 
the 148Glu allele and cancer risk (reviewed in ref. 10). On the contrary, Hu et al have demonstrated that the variant form of the enzyme is associated with cell cycle G2 delay in response to IR, therefore contributing to IR hypersensitivity (17). Also, the APEX1 Asp148Glu polymorphism was not shown to be associated with the non-medullary form of TC (25) in the only association study concerning TC that came to our knowledge, nor was it associated with thyroid nodules in a well-powered study involving IR-exposed populations (28). In agreement with this overwhelming evidence, our results do not support an association between APEX1 Asp148Glu polymorphism and $\mathrm{TC}$ risk, since neither the differences in genotypic frequencies observed between case and control groups nor ORs are statistically significant.

PARP1 is an abundant nuclear protein that can bind to DNA and promote the poly(ADP-ribosyl)ation of a variety of proteins (including itself). Among other roles, PARP1 has a major signalling role in DNA damage detection and repair, acting as a molecular nick sensor to initiate the recruitment of XRCC1 and other DNA repair proteins: transient binding of PARP1 to DNA single or double strand breaks allows for autoribosylation and subsequent interaction with XRCC1 and, possibly, a number of other DNA repair proteins (44). One common non-synonymous SNP, PARPI Val762Ala, results in an amino acid substitution within the $\mathrm{COOH}$-terminal catalytic domain of the enzyme. This variant has been associated with reduced enzymatic activity (44) and limited capacity for interaction with XRCC1 (45). This may result in attenuated BER capacity and thus increased cancer predisposition in $P A R P 1$ Ala762 carriers. In fact, the variant form of the PARPI Val762Ala polymorphism has been associated, in several wellpowered clinical association studies, with increased cancer susceptibility, namely lung (44) and GI tract (45) cancers. Studies regarding other types of cancer, namely breast cancer $(22,46)$, have failed to demonstrate an association between PARPI Val762Ala genotype and cancer susceptibility. The results obtained in this study do not suggest any association between PARPI Val762Ala polymorphism and TC risk (Table V), in agreement with the results reported by Chiang et al (25) in the only other clinical association study where the role of PARPI Val762Ala polymorphism on TC risk was assessed, suggesting that this polymorphism may not play a significant role in the disease. However, given the evidence available and the possibility for interaction with smoking status and/or XRCC1 genotype $(25,44,45)$, more in depth studies should be pursued in order to further clarify this issue.

Concluding, our results do not reveal a significant involvement of XRCC1 Arg194Trp and Arg399Gln, OGG1 Ser326Cys, APEX1 Asp148Glu, MUTYH Gln335His and PARP1 Val762Ala polymorphisms on the individual susceptibility towards TC, mostly in aggreement with the limited evidence that is available specifically for TC risk. This was the first time that the putative association between the MUTYH Gln335His polymorphism and TC susceptibility was evaluated: when the histological stratification analysis was performed, we observed that the association between the presence of heterozygozity in the MUTYH Gln335His polymorphism and TC risk almost reached significance for the papillary subtype of TC. Since the sample size was modest, the possibility of a type I error should not be excluded and this result should, therefore, be interpreted with caution. More in depth studies involving larger populations should be pursued in order to further clarify this issue and to verify the potential usefulness of the MUTYH Gln335His genotype as a predictive biomarker of susceptibility to TC. Also, since BER genetic variation alone seems to have, at best, only a modest impact on TC susceptibility (unless when considered together with other relevant risk factors), future studies regarding the putative role of BER polymorphisms on TC risk should be powered to allow for the study of gene-environment (e.g. smoking and drinking status, IR exposure) and gene-gene (e.g. other DNA repair or chemical metabolizing enzymes) interactions as well as stratified analysis according to histological subtype and disease developmental stage. Haplotype analysis should also be considered.

\section{Acknowledgements}

We wish to thank Luísa Manso Oliveira, Lylliane Luz, Silvia Morgado Amaro and Maria Catarina Soveral for technical support. This study was supported by the Center for Research in Human Molecular Genetics (CIGMH), Projects PTDC/ SAU-OSM/105572/2008, PTDC/SAU-ESA/102367/2008 and PTDC/QUI/67522/2006 from Fundação para a Ciência e Tecnologia (FCT) and Fundação Calouste Gulbenkian (Grant 76438/2006). The grants to M. Pingarilho (SFRH/ $\mathrm{BD} / 22612 / 2005$ ) from FCT are also acknowledged.

\section{References}

1. De Lellis RA and Williams ED: Tumours of the thyroid and parathyroid. In: Pathology and Genetics of Tumours of Endocrine Organs. De Lellis RA, Lloyd RV, Heitz PU and Eng C (eds). IARC Press, Lyon, pp51-56, 2004.

2. Dal Maso L, Bosetti C, La Vecchia C and Franceschi S: Risk factors for thyroid cancer: an epidemiological review focused on nutritional factors. Cancer Causes Control 20: 75-86, 2009.

3. Landa I and Robledo M: Association studies in thyroid cancer susceptibility: are we on the right track? J Mol Endocrinol 47: R43-R58, 2011.

4. Sarasin A, Bounacer A, Lepage F, Schlumberger M and Suarez H: Mechanisms of mutagenesis in mammalian cells. Application to human thyroid tumours. C R Acad Sci III 322: 143-149, 1999.

5. Adjadj E, Schlumberger M and de Vathaire F: Germ-line DNA polymorphisms and susceptibility to differentiated thyroid cancer. Lancet Oncol 10: 181-190, 2009.

6. Gudmundsson J, Sulem P, Gudbjartsson DF, et al: Common variants on $9 \mathrm{q} 22.33$ and $14 \mathrm{q} 13.3$ predispose to thyroid cancer in European populations. Nat Genet 41: 460-464, 2009.

7. Hoeijmakers JHJ: Genome maintenance mechanisms for preventing cancer. Nature 411: 366-374, 2001.

8. Sigurdson AJ, Hauptmann M, Alexander BH, Doody MM, Thomas CB, Struewing JP and Jones IM: DNA damage among thyroid cancer and multiple cancer cases, controls, and long-lived individuals. Mutat Res 586: 173-188, 2005.

9. Gatzidou E, Michailidi C, Tseleni-Balafouta S and Theocharis S: An epitome of DNA repair related genes and mechanisms in thyroid carcinoma. Cancer Lett 290: 139-147, 2010.

10. Wilson DM III, Kim D, Berquist BR and Sigurdson AJ: Variation in base excision repair capacity. Mutat Res 711: 100-112, 2011.

11. Hung RJ, Hall J, Brennan P and Boffetta P: Genetic polymorphisms in the base excision repair pathway and cancer risk: a HuGE review. Am J Epidemiol 162: 925-942, 2005.

12. Silva SN, Gil OM, Oliveira VC, et al: Association of polymorphisms in ERCC2 gene with non-familial thyroid cancer risk. Cancer Epidemiol Biomarkers Prev 14: 2407-2412, 2005.

13. Gomes BC, Silva SN, Azevedo AP, et al: The role of common variants of non-homologous end-joining repair genes XRCC4, LIG4 and Ku80 in thyroid cancer risk. Oncol Rep 24: 1079-1085, 2010. 
14. Bastos HN, Antão MR, Silva SN, et al: Association of polymorphisms in genes of the homologous recombination DNA repair pathway and thyroid cancer risk. Thyroid 19: 1067-1075, 2009.

15. Xu L, Doan PC, Wei Q, Liu Y, Li G and Sturgis EM: Association of BRCA1 functional single nucleotide polymorphisms with risk of differentiated thyroid carcinomaa. Thyroid 22: 35-43, 2012.

16. Silva SN, Gomes BC, Rueff J and Gaspar JF: DNA repair perspectives in thyroid and breast cancer: the role of DNA repair polymorphisms. In: DNA Repair and Human Health. Vengrova S (ed). InTech, pp459-484, 2011.

17. Hu JJ, Smith TR, Miller MS, Mohrenweiser HW, Golden A and Case LD: Amino acid substitution variants of APE1 and $\mathrm{XRCC1}$ genes associated with ionizing radiation sensitivity. Carcinogenesis 22: 917-922, 2001.

18. Vodicka P, Stetina R, Polakova V, et al: Association of DNA repair polymorphisms with DNA repair functional outcomes in healthy human subjects. Carcinogenesis 28: 657-664, 2007.

19. Silva SN, Moita R, Azevedo AP, et al: Menopausal age and $\mathrm{XRCC1}$ gene polymorphisms: role in breast cancer risk. Cancer Detect Prev 31: 303-309, 2007.

20. Lange K, Cantor R, Horvath S, Perola M, Sabatti C, Sinsheimer J and Sobel E: Mendel version 4.0: a complete package for the exact genetic analysis of discrete traits in pedigree and population data sets. Am J Hum Genet 69: A1886, 2001.

21. Kiuru A, Lindholm C, Heinavaara S, et al: XRCC1 and XRCC3 variants and risk of glioma and meningioma. J Neurooncol 88 : $135-142,2008$

22. Zhang Y, Newcomb PA, Egan KM, et al: Genetic polymorphisms in base-excision repair pathway genes and risk of breast cancer Cancer Epidemiol Biomarkers Prev 15: 353-358, 2006.

23. Conde J, Silva S, Azevedo A, Teixeira V, Pina J, Rueff J and Gaspar J: Association of common variants in mismatch repair genes and breast cancer susceptibility: a multigene study. BMC Cancer 9: 344, 2009.

24. Ginsberg G, Angle K, Guyton K and Sonawane B: Polymorphism in the DNA repair enzyme XRCC1: utility of current database and implications for human health risk assessment. Mutat Res 727: 1-15, 2011.

25. Chiang FY, Wu CW, Hsiao PJ, et al: Association between polymorphisms in DNA base excision repair genes XRCC1, APE1, and ADPRT and differentiated thyroid carcinoma. Clin Cancer Res 14: 5919-5924, 2008.

26. García-Quispes WA, Pérez-Machado G, Akdi A, et al: Association studies of OGG1, XRCC1, XRCC2 and XRCC3 polymorphisms with differentiated thyroid cancer. Mutat Res 709-710: 67-72, 2011.

27. Ryu RA, Tae K, Min HJ, Jeong JH, Cho SH, Lee SH and Ahn YH: $\mathrm{XRCCl}$ polymorphisms and risk of papillary thyroid carcinoma in a Korean sample. J Korean Med Sci 26: 991-995, 2011.

28. Sigurdson AJ, Land CE, Bhatti P, et al: Thyroid nodules, polymorphic variants in DNA repair and RET-related genes, and interaction with ionizing radiation exposure from nuclear tests in Kazakhstan. Radiat Res 171: 77-88, 2009.

29. Ho T, Li G, Lu J, Zhao C, Wei Q and Sturgis EM: Association of XRCC1 polymorphisms and risk of differentiated thyroid carcinoma: a case-control analysis. Thyroid 19: 129-135, 2009.

30. Akulevich NM, Saenko VA, Rogounovitch TI, et al: Polymorphisms of DNA damage response genes in radiationrelated and sporadic papillary thyroid carcinoma. Endocr Relat Cancer 16: 491-503, 2009.
31. Wei B, Zhou Y, Xu Z, et al: The effect of hOGG1 Ser326Cys polymorphism on cancer risk: evidence from a meta-analysis. PLoS One 6: e27545, 2011.

32. Gu D, Wang M, Zhang Z and Chen J: Lack of association between the hOGG1 Ser326Cys polymorphism and breast cancer risk: evidence from 11 case-control studies. Breast Cancer Res Treat 122: 527-531, 2010

33. Yuan W, Xu L, Feng Y, et al: The hOGG1 Ser326Cys polymorphism and breast cancer risk: a meta-analysis. Breast Cancer Res Treat 122: 835-842, 2010.

34. David SS, O'Shea VL and Kundu S: Base-excision repair of oxidative DNA damage. Nature 447: 941-950, 2007.

35. Cheadle JP and Sampson JR: MUTYH-associated polyposis from defect in base excision repair to clinical genetic testing. DNA Repair 6: 274-279, 2007.

36. Aretz S, Uhlhaas S, Goergens H, et al: MUTYH-associated polyposis: 70 of 71 patients with biallelic mutations present with an attenuated or atypical phenotype. Int J Cancer 119: 807-814, 2006.

37. Pervaiz M, Eppolito A and Schmidt K: Papillary thyroid cancer in a patient with MUTYH-associated polyposis (MAP). Fam Cancer 9: 595-597, 2010.

38. Ali M, Kim H, Cleary S, Cupples C, Gallinger S and Bristow R: Characterization of mutant MUTYH proteins associated with familial colorectal cancer. Gastroenterology 135: 499-507, 2008.

39. Picelli S, Zajac P, Zhou XL, et al: Common variants in human CRC genes as low-risk alleles. Eur J Cancer 46: 1041-1048, 2010.

40. Tao H, Shinmura K, Suzuki M, et al: Association between genetic polymorphisms of the base excision repair gene MUTYH and increased colorectal cancer risk in a Japanese population. Cancer Sci 99: 355-360, 2008

41. Miyaishi A, Osawa K, Osawa Y, et al: MUTYH Gln324His gene polymorphism and genetic susceptibility for lung cancer in a Japanese population. J Exp Clin Cancer Res 28: 10, 2009.

42. Qian B, Zhang H, Zhang L, Zhou X, Yu H and Chen K: Association of genetic polymorphisms in DNA repair pathway genes with non-small cell lung cancer risk. Lung Cancer 73: 138-146, 2011.

43. Huang M, Dinney CP, Lin X, Lin J, Grossman HB and Wu X: High-order interactions among genetic variants in DNA base excision repair pathway genes and smoking in bladder cancer susceptibility. Cancer Epidemiol Biomarkers Prev 16: 84-91, 2007.

44. Jiang J, Zhang X, Yang H and Wang W: Polymorphisms of DNA repair genes: ADPRT, XRCC1, and XPD and cancer risk in genetic epidemiology. Methods Mol Biol 471: 305-333, 2009.

45. Miao X, Zhang X, Zhang L, et al: Adenosine diphosphate ribosyl transferase and X-ray repair cross-complementing 1 polymorphisms in gastric cardia cancer. Gastroenterology 131: 420-427, 2006.

46. Zhai X, Liu J, Hu Z, et al: Polymorphisms of ADPRT Val762Ala and XRCC1 Arg399Glu and risk of breast cancer in Chinese women: a case control analysis. Oncol Rep 15: 247-252, 2006. 